\title{
EVALUACIÓN DE ESTILOS DE VIDA SALUDABLE EN LA FACULTAD DE MEDICINA DE LA FUNDACIÓN UNIVERSITARIA DE CIENCIAS DE LA SALUD*
}

\author{
Silvia Lorena Mora MD**, Fabio Alberto Múnera MD***
}

\section{Resumen}

La política de universidad saludable se basa en la promoción de la salud para brindar una mejor calidad de vida, que propicia el desarrollo humano y promueve la formación de individuos que actúan como modelo en conductas saludables frente a su entorno familiar, laboral y social. Objetivo: evaluar los estilos de vida saludables en la población de la Facultad de Medicina de la Fundación Universitaria de Ciencias de la Salud, Bogotá DC, Colombia. Materiales y métodos: estudio de corte transversal. Se utilizó una encuesta propuesta por la OPS que cuenta con 10 dominios y 45 ítems, el diligenciamiento se hizo por correo electrónico. Resultados: en el primer semestre de 2012 se invitó a participar a una población de 431 individuos, la tasa de respuesta fue de $82 \%$ (n 355), 39.7\% hombres. El $72 \%$ obtuvo $>70$ puntos lo que correspondería a buenos hábitos de vida saludable y el $0.6 \%$ uno de $\leq 40$ indicando que no los tenían. El $76 \%$ no realizaba actividad física, $46 \%$ no tenía adecuado descanso y $\mathbf{3 0 . 8 \%}$ autorreportó malos hábitos nutricionales. Conclusión: aunque la escala clasifica a más del $70 \%$ de la población con hábitos saludables, fueron inadecuados los estilos de vida relacionados con actividad física, nutrición y descanso. Se deben generar estrategias de intervención colectiva que permitan modificar los factores de riesgo.

Palabras clave: hábitos; descanso, nutrición, sexualidad, encuestas nutricionales, actividad física o motora.

\section{EVALUATION OF HEALTHY LIFESTYLES AT THE SCHOOL OF MEDICINE OF FUNDACIÓN UNIVERSITARIA DE CIENCIAS DE}

\section{LA SALUD}

\section{Abstract}

The healthy universities policy is based on health promotion to offer a better quality of life favoring human development forming individuals to serve as role models of healthy behaviors in their family, work and social settings. Objective: to assess practices regarding healthy lifestyles among medical students of Fundación Universitaria de Ciencias de la Salud, Bogotá DC, Colombia. Materials and Methods: a cross-sectional study applying a survey developed by the PAHO consisting of 10 domains and 45 items answered by email. Results: In the first semester of 2012 a population of 431 individuals was invited to participate, the response rate was $82 \%$ (n 355), 39.7\% were men. As $72 \%$ obtained $>70$ points this would indicate the proportion that practiced good habits of healthy lifestyles and $0.6 \%, 0$ one of $\leq 40$, indicated those who did not, $76 \%$ did not practice physical activity, $46 \%$ did not have healthy rest habits and $30.8 \%$ self-reported poor nutritional habits. Conclusion: Although the scale classifies more than $\mathbf{7 0 \%}$ of the population as practicing healthy habits, the lifestyles regarding physical activity, nutrition and rest were inadequate. Collective intervention strategies which allow risk factor modification must be developed.

Key words: Habits; rest, nutrition, sexuality, nutrition surveys, physical or gross motor activity

Fecha recibido: marzo 24 de 2015 - Fecha aceptado: mayo 29 de 2015

* Bogotá DC, Colombia.

** Especialista en Medicina Familiar, Profesor Asociado Facultad de Medicina, Fundación Universitaria de Ciencias de la Salud, Bogotá DC, Colombia.
*** Residente III año de Medicina Familiar, Fundación Universitaria de Ciencias de la Salud, Bogotá DC, Colombia. 


\section{Introducción}

Se define universidad saludable como aquella institución que se basa en el concepto de promoción de la salud como herramienta para brindar a sus integrantes una mejor calidad de vida, que propicia su desarrollo humano, y que promueve la formación de individuos que actúan como modelo de conductas saludables frente a su entorno familiar, laboral y social. ${ }^{1}$ El establecimiento de instituciones de educación superior como promotoras de la salud comenzó en la red europea, que llevó a cabo su primera conferencia internacional en Lacaster (Reino Unido) en 1999. ${ }^{1}$ Después en octubre de 2005 durante la Segunda Conferencia Internacional de la Red Europea de Universidades Promotoras de la Salud, se emitió la Carta de Edmonton, que constituye un documento en el cual se especifica el papel de las instituciones de educación superior como entidades encargadas de la promoción de la salud.

En Chile las primeras nociones del tema se iniciaron en el año 1999 cuando incorporaron el programa a instituciones de pre y posgrado, luego de convenios tecnológicos con el centro de promoción de salud de la Universidad de Toronto Canadá. La Primera Conferencia Internacional de Universidades Promotoras de Salud se desarrolló en Santiago de Chile en noviembre de 2003. ${ }^{2}$ En Colombia la primera institución que inició estudios para la implementación de esta política fue la Pontificia Universidad Javeriana hacia 2003. ${ }^{2}$ Universidad saludable es considerada una política institucional en algunos centros de educación superior a nivel mundial; es por esto que el área de medicina familiar de la Fundación Universitaria de Ciencias de la Salud (FUCS) inició la fase diagnóstica de universidad saludable como proyecto de investigación en 2009.

\section{Materiales y métodos}

Se realizó un estudio de corte transversal en la Facultad de Medicina de la FUCS invitando a participar al personal docente, administrativo y estudiantes de pre y posgrado. Los criterios de inclusión fueron todos los estudiantes desde el segundo semestre hasta internado, los de posgrado de las diferentes áreas quirúrgicas y clínicas, los docentes y el personal administrativo. Los criterios de exclusión fueron estudiantes de diplomados y otros cursos de educación médica continuada, así como pasantes, estudiantes de intercambio o en procesos de homologación de materias. A partir de listados de estudiantes, personal docente y administrativo se realizó un muestreo aleatorio simple con asignación proporcional, se estimó un error tipo 1 del 5\% de una población total de 1104 individuos. Se realizaron ajustes por falta de respuesta del $20 \%$, obteniendo un tamaño de muestra de 431.

La encuesta ¿cómo es mi estilo de vida? propuesta por la OPS, adaptada por la Pontificia Universidad Javeriana (suministrada por la doctora Constanza Granados coordinadora de ese programa en su proceso de estudio e implementación de universidad saludable), fue utilizada para este estudio con el fin de generar la línea de base y así determinar los estilos de vida saludable en la facultad de medicina de la FUCS. El instrumento consta de 10 dominios (45 ítems): 1) relación con otros, 2) actividad física, 3) descanso, 4) nutrición, 5) salud oral, 6) sexualidad, 7) movilidad, 8) consumo de sustancias, 9) sentido de vida y 10) medio ambiente. La calificación designada a cada uno de los ítems se reporta en una escala ordinal siendo $0=$ nunca o casi nunca, $1=$ a veces y $2=$ siempre/casi siempre. $\mathrm{La}$ puntuación que califica si existen hábitos de vida saludable es: $\angle 40=$ "tus estilos de vida te ponen en riesgo, tu salud es tu mayor valor, piensa cambios que quieras lograr, ponte metas pequeñas y busca orientación para mantener tu salud"; 41-58= "en el cuidado de tu salud tienes estilos de vida que te protegen pero otros que te ponen en riesgo, es el momento para hacer cambios a tu favor es aquí y ahora"; 59-69= "adecuado, estás bien pero puedes mejorar en donde los puntajes te muestran algún riesgo"; y 70-80= tienes estilos de vida que te ayudarán a mantenerte saludable" (Tabla 1).

La forma de aplicación de esta encuesta fue mediante el envío a los correos electrónicos tanto personales como institucionales de cada uno de los seleccionados, con previa invitación y haciendo énfasis en la privacidad de sus respuestas. Se enviaron cada dos días y se les solicitaba que si ya lo habían diligenciado, omitieran la invitación para evitar doble respuesta. El periodo de recolección de la muestra fue desde el $1^{\circ}$ 


\begin{tabular}{|l|l|}
\multicolumn{2}{|c|}{ Tabla I. Rangos de calificación de los estilos } \\
de vida saludables \\
de 70 a 80 & $\begin{array}{l}\text { Felicitaciones tienes estilos de vida que te ayuda- } \\
\text { rán a mantenerte saludable. }\end{array}$ \\
\hline de 59 a 69 & $\begin{array}{l}\text { Adecuado, estás bien pero puedes mejorar en } \\
\text { donde los puntajes te muestran algún riesgo. }\end{array}$ \\
\hline de 41 a 58 & $\begin{array}{l}\text { En el cuidado de tu salud tienes estilos de vida que } \\
\text { te protegen pero otros que te ponen en riesgo, el } \\
\text { momento para hacer cambios a tu favor es aquí } \\
\text { y ahora. }\end{array}$ \\
\hline >de 40 & $\begin{array}{l}\text { Tus estilos de vida te ponen en riesgo, tu salud es } \\
\text { tu mayor valor, piensa cambios que quieras lograr, } \\
\text { ponte metas pequeñas y busca orientación para } \\
\text { mantener tu salud. }\end{array}$ \\
\hline
\end{tabular}

de febrero hasta el 29 de abril de 2012. El protocolo fue aprobado por el comité de investigaciones de la facultad de medicina y el comité de ética médica clasificándola como investigación sin riesgo. Los resultados de la escala se resumieron con frecuencias absolutas y porcentajes según el tipo de usuario; el análisis estadístico se realizó en stata 10.

\section{Resultados}

La población invitada a participar en el estudio fue de 431 miembros de la facultad de medicina; el total de respuesta fue 355 (82\%), no retornando la encues- ta diligenciada 76 (18\%) (Tabla 2). Por género 214 (49.6\%) fueron mujeres y $217(50.3 \%)$ hombres. De ellos, $72.2 \%$ con un IC 95\%, con 68 a $73.3 \%$ que autorreportó buenos estilos de vida saludable; $19.2 \%$ con un IC del 95\%, de los cuales no correspondió a estilos de vida aceptable; $7.6 \%$ con IC $95 \%$, con 4.8 a $10.3 \%$ que informó algunos hábitos saludables protectores pero otros que no lo eran; y $0.6 \%$ con un IC $95 \%$, de quienes 0 a $1.3 \%$ no tenían buenos hábitos de vida saludable. ${ }^{3}$

Al evaluar los estilos de vida saludable de acuerdo con el género se encontró que en el grupo de las mujeres $0.9 \%$ no tenía hábitos saludables, en tanto que los hombres no puntuaron en este rango. El $6.07 \%$ de las encuestadas y el $9.9 \%$ de los hombres, poseían algunos estilos de vida saludable, pero otros no. El 20.6\% de las mujeres y $17 \%$ de los hombres tenían estilos de vida adecuados y $72.4 \%$ de las mujeres y $73 \%$ de los hombres tenían estilos de vida saludable (Tabla 3). Es de destacar que $82.8 \%$ de los docentes, $70 \%$ de los estudiantes de pregrado, $74 \%$ de estudiantes de posgrado y $93 \%$ del personal administrativo obtuvieron puntajes 70 , esto los califica que "tenían estilos de vida que les ayudarían a mantenerse saludables" (Tabla 3).

La actividad física dentro de los resultados analizados es la variable que más bajas puntuaciones presentó

\begin{tabular}{|c|c|c|c|c|c|c|}
\hline & Administrativo & Docente & Estudiante & Estudiante & Total & IC \\
\hline \multirow[t]{2}{*}{ Categoría } & n 15 & n 29 & $\begin{array}{c}\text { Posgrado } \\
\text { n } 50\end{array}$ & $\begin{array}{c}\text { Pregrado } \\
\text { n } 261\end{array}$ & n 355 & IC $95 \%$ \\
\hline & n (\%) & n (\%) & n (\%) & n (\%) & n (\%) & \\
\hline$<40^{*}$ & - & - & - & $2(0.7)$ & $2(0.6)$ & $0.6(0-1.3)$ \\
\hline 41-58t & - & $1(3.4)$ & $5(10)$ & $21(8)$ & $27(7.6)$ & $7.6(4.810 .3)$ \\
\hline $59-69 \ddagger$ & $1(6.7)$ & $4(13.8)$ & $8(16)$ & $55(21.1)$ & $68(19.2)$ & $19.2(15-23)$ \\
\hline$\geq 709$ & 14(93.3) & $24(82.8$ & $37(74)$ & $183(70.1)$ & $258(72.7)$ & $72.7(68-73)$ \\
\hline
\end{tabular}

$*<40$. estilos de vida que ponen en riesgo la salud; $\nmid 4 \mathrm{I}-58$; estilos de vida que protegen pero otros no; $\ddagger$ adecuados estilos de vida; II buenos estilos de vida saludable. 


\begin{tabular}{|c|c|c|c|c|}
\hline \multirow{2}{*}{ Puntaje total } & \multicolumn{2}{|c|}{ Mujeres } & \multicolumn{2}{|c|}{ Hombres } \\
\hline & $\mathbf{N}$ & (\%) & $\mathbf{N}$ & (\%) \\
\hline$<40$ & 2 & $(0.9)$ & 0 & $(0)$ \\
\hline $41-58$ & 13 & (6.0) & 14 & (9.9) \\
\hline $59-69$ & 44 & (20.5) & 24 & (17) \\
\hline$\geq 70$ & 155 & (72.4) & 103 & (73) \\
\hline Total & 214 & (100) & 141 & (100) \\
\hline
\end{tabular}

en los cuatro grupos encuestados, pues $60.5 \%$ de la población expresó no tener una buena actividad física; se destaca, por el contrario, que el de los estudiantes de pregrado es el que más realiza ya que el $22.6 \%$ afirma hacerla. En las preguntas que evalúan el desempeño se observó como a la pregunta 1.2, que hace alusión a "realizó actividad física al menos 30 minutos, cinco días a la semana" el $62.8 \%$ de docentes, $56 \%$ de estudiantes de pregrado, $84 \%$ de posgrado y $60 \%$ del personal administrativo manifestaron "nunca"; de igual forma para la pregunta 1.1 "realizó actividad física diaria con una intensidad moderada (caminar, bailar, trotar) por lo menos 30 minutos", el $37.9 \%$ de docentes, $40.6 \%$ de estudiantes de pregrado, $54 \%$ de posgrado y $53 \%$ del personal administrativo dieron como respuesta que "nunca" (Tabla 4).

En el dominio que evalúa el descanso se pudo apreciar como en la pregunta 3.3 "disfruto mi tiempo libre", el $27.5 \%$ de docentes, $36.7 \%$ de estudiantes de pregrado, $36 \%$ de posgrado y $53 \%$ del personal administrativo afirmó que "nunca" disfrutaba de su tiempo libre (Tabla 4).

Entre las preguntas que evalúan la nutrición se encontró que para el ítem 4.4 "me siento a gusto con mi peso", el $27.5 \%$ de docentes, $24.5 \%$ de estudiantes de pregrado, $24 \%$ de posgrado y $20 \%$ del personal administrativo expresó que no se sentían a gusto (Tabla 4).

Los dominios que mejor evaluación obtuvieron fueron: sexualidad, movilidad y consumo de sustancias; es así

\begin{tabular}{|c|c|c|c|c|c|}
\hline Dominio & \multicolumn{5}{|c|}{ Resultados porcentuales } \\
\hline \multirow{6}{*}{$\begin{array}{l}\text { Actividad } \\
\text { física }\end{array}$} & \multicolumn{5}{|c|}{$\begin{array}{l}\text { Ítem I.I "Nunca" realiza actividad física diaria con una intensidad moderada (caminar, bailar, trotar) } \\
\text { por lo menos } 30 \text { minutos }\end{array}$} \\
\hline & Docentes & $\begin{array}{l}\text { Estudiantes de } \\
\text { pregrado }\end{array}$ & $\begin{array}{l}\text { Estudiantes de } \\
\text { posgrado }\end{array}$ & Administrativos & Total \\
\hline & $38 \%$ & $40,6 \%$ & $54 \%$ & $53 \%$ & $60,5 \%$ \\
\hline & \multicolumn{5}{|c|}{ Ítem I.2 “Nunca” realiza actividad física al menos 30 minutos cinco días a la semana. } \\
\hline & Docentes & $\begin{array}{l}\text { Estudiantes de } \\
\text { pregrado }\end{array}$ & $\begin{array}{l}\text { Estudiantes de } \\
\text { posgrado }\end{array}$ & Administrativos & Total \\
\hline & $62,8 \%$ & $56 \%$ & $84 \%$ & $60 \%$ & $43 \%$ \\
\hline \multirow{3}{*}{ Descanso } & \multicolumn{5}{|c|}{ Ítem 3.3 “Nunca” disfruto mi tiempo libre } \\
\hline & Docentes & $\begin{array}{l}\text { Estudiantes de } \\
\text { pregrado }\end{array}$ & $\begin{array}{l}\text { Estudiantes de } \\
\text { posgrado }\end{array}$ & Administrativos & Total \\
\hline & $27,5 \%$ & $36,7 \%$ & $36 \%$ & $53 \%$ & $36,6 \%$ \\
\hline \multirow{3}{*}{ Nutrición } & \multicolumn{5}{|c|}{ ITEM 4.4 “nunca” me siento a gusto con mi peso. } \\
\hline & Docentes & $\begin{array}{l}\text { Estudiantes de } \\
\text { pregrado }\end{array}$ & $\begin{array}{l}\text { Estudiantes de } \\
\text { posgrado }\end{array}$ & Administrativos & Total \\
\hline & $27,5 \%$ & $24,5 \%$ & $24 \%$ & $20 \%$ & $24,5 \%$ \\
\hline
\end{tabular}




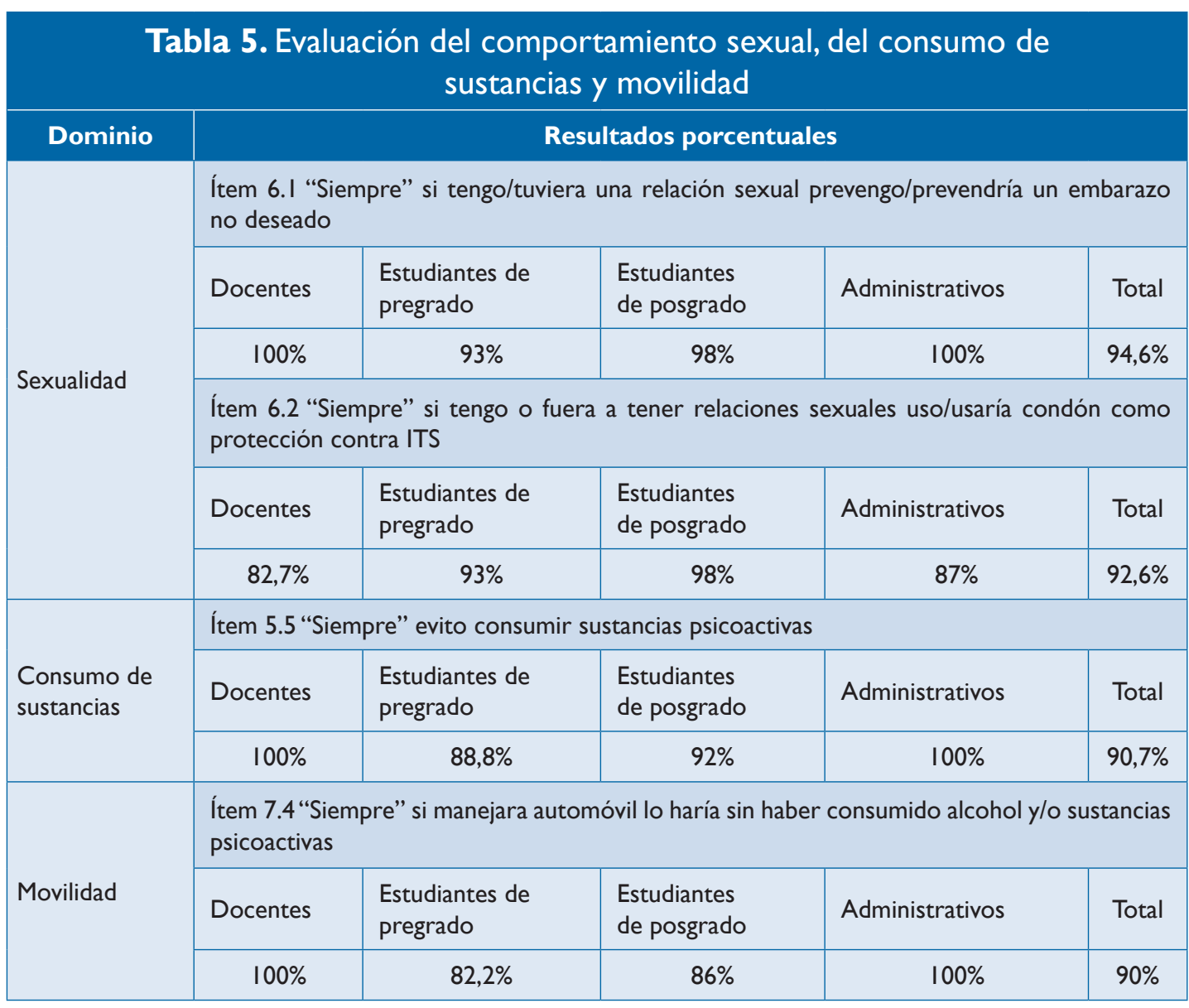

como el relativo a sexualidad y en particular los ítem 6.1 "si tengo/tuviera una relación sexual prevengo/ prevendría un embarazo no deseado", el 100\% de los docentes, $93 \%$ de los estudiantes de pregrado, $98 \%$ de posgrado y $100 \%$ de administrativos manifestaron que "siempre" lo prevendrían. El ítem 6.2 "si tengo o fuera a tener relaciones sexuales uso o usaría condón como protección contra ITS", el $82.7 \%$ de docentes, 93\% de estudiantes de pregrado, $98 \%$ de posgrado y $86.6 \%$ de administrativos, afirmaron que "siempre" lo usarían (Tabla 5).

El dominio que hace referencia al consumo de sustancias y en especial el ítem 8.5 "evito consumir sustancias psicoactivas", el $100 \%$ de los docentes, $88.8 \%$ de estudiantes de pregrado, $92 \%$ de posgrado y $100 \%$ del personal administrativo revelaron que "siempre" lo evitaban (Tabla 5).

La movilidad fue otro de los dominios evaluados por el grupo investigador, haciendo referencia en parti- cular al ítem 7.4 "si manejara automóvil, lo haría sin haber consumido alcohol y/o sustancias psicoactivas" encontrando que el $100 \%$ de docentes, $82.2 \%$ de estudiantes de pregrado, $86 \%$ de posgrado y $100 \%$ de los administrativos afirmaron que "siempre" lo evitaban (Tabla 5).

\section{Discusión}

Los estilos de vida saludable son aquellas conductas o comportamientos, tanto individuales como colectivos, con los cuales alcanzamos un bienestar no solo físico sino también emocional, que se reflejará en un adecuado estado de salud que mejora la calidad de vida. Para entender y poder aplicar a este concepto debemos partir de la base de que existen factores de riesgo como también protectores, los cuales al interactuar en forma dinámica en los individuos, permitirán la expresión de la enfermedad o la ausencia de la misma. ${ }^{3}$ 
Recordemos que existen factores de riesgo modificables y no modificables, por lo tanto estamos llamados a realizar actividades educativas y por ende preventivas con el fin de intervenir en forma oportuna sobre los factores que son susceptibles de modificar y de esta forma evitar o al menos retardar la manifestación de los estados mórbidos que deterioran la calidad de vida.

Es de anotar que el presente estudio se comporta como una prueba piloto, la que después se pretende aplicar en cada una de las facultades de la FUCS; con el análisis de los resultados arrojados en cada una ellas, se tendrá una línea de base que permitirá implementar los hábitos saludables como forma de vida en cada uno de los integrantes de la comunidad educativa (Tabla 6).

Dentro de las limitaciones evidenciadas en la recolección de la información estuvo la dificultad de eliminar las dobles respuestas al cuestionario, pues teniendo en cuenta el principio de preservar la confidencialidad de los participantes, no se podía detectar el usuario que lo diligenció en más de una oportunidad. Una limitante digna de mencionar es que no se tuvieron en cuenta otras variables, entre ellas la edad, lo que limitó las interpretaciones de los comportamientos por grupos de edad.

Es importante hacer mención que una desventaja del instrumento empleado y su forma de ejecución es la facilidad de cambiar respuestas por parte de los entrevistados y de esta forma podían mejorar su autopercepción en algunos estilos de vida saludable; si bien es cierto el estudio fue confidencial y anónimo, al enfrentarse ante preguntas que exploraban su práctica sexual y el consumo de bebidas alcohólicas, entre otras, se podía generar un autocuestionamiento que impulsara a mejorar las calificaciones en algunos ítem específicos.

A pesar de que el $70 \%$ de la población participante de la Facultad de Medicina reportó que tenía hábitos de autocuidado, llama la atención que los ítems de actividad física, nutrición y descanso contradicen la autovaloración en la población estudiada, esto daría elementos para reevaluar el instrumento empleado con el fin de realizar cambios tendientes a mejorar su diseño y por ende su aplicación para obtener valores más ajustados a la realidad.

En cuanto a la actividad física, se pudo observar que en los grupos de docentes, administrativos, estudiantes de pre y posgrado se aseveró que, en su mayoría, tenían poca actividad física. Por otra parte, se pudo inferir que los resultados obtenidos al compararlos con los de la población participante en el estudio de la Encuesta Nacional de la Situación Nutricional en Colombia (ENSIN 2010), no distan de la realidad nacional, ya que en esta solo una de cada tres personas realizaba actividad física (caminar) como medio de trasporte, una de cada cinco hacía actividad física en su tiempo libre y solo una de cada dieciocho montaba en bicicleta. ${ }^{4}$

En el estudio "Prevalencia de los factores de riesgo cardiometabólico en la población de estudiantes de posgrado y docentes de la facultad de medicina de la Fundación Universitaria de Ciencias de la Salud 2010" realizado por los doctores José Fernando Orduz, Juan Carlos Ospina y Dairo Beltrán ${ }^{5}$, reportó un porcentaje de sedentarismo de $71,7 \%$ en los docentes y del $84.5 \%$ en los estudiantes de posgrado. Tanto en el estudio citado como en el nuestro, pese a la diferencia de dos años en sus ejecuciones, se encontraron resultados muy similares en los dos grupos encuestados en lo que respecta a la baja práctica de actividad física.

En relación con la nutrición se evidenció en los cuatro grupos encuestados que calificaban en forma inadecuada las prácticas nutricionales. Haciendo referencia al ítem que evalúa la "satisfacción con el peso", se demostró un alto inconformismo en la población encuestada, este hecho se ve reflejado de igual forma en la percepción de un peso inadecuado por parte de población colombiana en el estudio (ENSIN 2010) ${ }^{4}$, ya que en este se concluyó que las personas con un nivel de educación superior a la secundaria presentaron mayores tendencias a sobreestimar su incremento en el IMC $(9,1 \%)$ comparadas con el 3,2\% de las que tenían estudios de primaria básica. 
Tabla 6. Encuesta OPS, adaptada por la Pontificia Universidad Javeriana ¿Cómo es mi estilo de vida?

\begin{tabular}{|c|c|c|c|c|c|c|}
\hline \multicolumn{4}{|r|}{ Califica cada frase en la columna respectiva: } & \multirow{2}{*}{$\begin{array}{c}\text { Nunca/Casi } \\
\text { nunca }\end{array}$} & \multirow{2}{*}{\begin{tabular}{|c|}
$\begin{array}{c}\text { A } \\
\text { veces }\end{array}$ \\
$\square$ \\
$\square$
\end{tabular}} & \multirow{2}{*}{\begin{tabular}{|c|}
$\begin{array}{c}\text { Siempre/Casi } \\
\text { siempre }\end{array}$ \\
$\square$
\end{tabular}} \\
\hline \multirow{7}{*}{ A } & \multirow{7}{*}{$\begin{array}{l}\text { Relaciones } \\
\text { con otros }\end{array}$} & 2.1 & Tengo con quien hablar de las cosas que son importantes para mi & & & \\
\hline & & 2.2 & Me siento valorado por otros & $\square$ & $\square$ & $\square$ \\
\hline & & 2.3 & Reconozco y valoro los aportes de los demás & $\square$ & $\square$ & $\square$ \\
\hline & & 2.4 & Realizo actividades que aportan a otros & $\square$ & $\square$ & $\square$ \\
\hline & & 2.5 & Disfruto estar con otras personas & $\square$ & $\square$ & $\square$ \\
\hline & & 2.6 & Siento que para otras personas es grato compartir conmigo & $\square$ & $\square$ & $\square$ \\
\hline & & 2.7 & $\begin{array}{l}\text { Me siento en capacidad de expresar respetuosamente las diferencias } \\
\text { a los demás }\end{array}$ & $\square$ & $\square$ & $\square$ \\
\hline \multirow{3}{*}{ B } & \multirow{3}{*}{$\begin{array}{l}\text { Actividad } \\
\text { física }\end{array}$} & 1.1 & \begin{tabular}{|l|l} 
Realizo actividad física diaria con una intensidad moderada \\
(caminar, bailar, trotar, etc.) por lo menos durante 30 minutos
\end{tabular} & $\square$ & $\square$ & $\square$ \\
\hline & & 1.2 & Realizo actividad física al menos 30 minutos, cinco días a la semana & $\square$ & $\square$ & $\square$ \\
\hline & & 1.3 & \begin{tabular}{|l|l} 
Tengo un rendimiento físico bueno \\
\end{tabular} & $\square$ & $\square$ & $\square$ \\
\hline \multirow{4}{*}{ C } & \multirow{4}{*}{ Descanso } & 3.1 & Duermo bien y me siento descansado & $\square$ & $\square$ & $\square$ \\
\hline & & 3.2 & Manejo el estrés y la tensión en mi vida & $\square$ & $\square$ & $\square$ \\
\hline & & 3.3 & Disfruto mi tiempo libre & $\square$ & $\square$ & $\square$ \\
\hline & & 3.4 & Encuentro alternativas interesantes para salir de la rutina & $\square$ & & \\
\hline \multirow{4}{*}{ D } & \multirow{4}{*}{ Nutrición } & 4.1 & $\begin{array}{l}\text { Balanceo mi alimentación frutas, verduras, proteínas, cereales y } \\
\text { lácteos }\end{array}$ & $\square$ & $\square$ & $\square$ \\
\hline & & 4.2 & $\begin{array}{l}\text { Evito consumir bebidas gaseosas, mucho azúcar o sal, comida chatarra } \\
\text { o con mucha grasa }\end{array}$ & $\square$ & $\square$ & $\square$ \\
\hline & & 4.3 & $\begin{array}{l}\text { En mi rutina diaria, destino un horario para tomar las comidas } \\
\text { principales (desayuno, almuerzo y comida) }\end{array}$ & $\square$ & $\square$ & $\square$ \\
\hline & & 4.4 & Me siento a gusto con mi peso actual & $\square$ & $\square$ & $\square$ \\
\hline \multirow{4}{*}{$\mathbf{E}$} & \multirow{4}{*}{ Salud oral } & 5.1 & $\begin{array}{l}\text { Considero que la salud oral es un elemento importante para } \\
\text { mantener mi salud general }\end{array}$ & $\square$ & $\square$ & $\square$ \\
\hline & & 5.2 & Cepillo mis dientes mínimo dos veces al día & $\square$ & $\square$ & $\square$ \\
\hline & & 5.3 & Uso además del cepillo y la crema, seda dental y enjuague bucal & $\square$ & $\square$ & $\square$ \\
\hline & & 5.4 & Visito al odontólogo por lo menos una vez al año & $\square$ & $\square$ & $\square$ \\
\hline \multirow{5}{*}{$\mathbf{F}$} & \multirow{5}{*}{ Sexualidad } & 6.1 & $\begin{array}{l}\text { Si tengo/tuviera una relación sexual prevengo/prevendría un } \\
\text { embarazo no deseado }\end{array}$ & $\square$ & $\square$ & $\square$ \\
\hline & & 6.2 & $\begin{array}{l}\text { Si tengo o fuera a tener relaciones sexuales, uso o usaría condón } \\
\text { como protección contra ITS (Infecciones de trasmisión sexual) }\end{array}$ & $\square$ & $\square$ & $\square$ \\
\hline & & 6.3 & $\begin{array}{l}\text { Evito/evitaría tener relaciones sexuales bajo el efecto del } \\
\text { alcohol o drogas }\end{array}$ & $\square$ & $\square$ & $\square$ \\
\hline & & 6.4 & Considero que mi sexualidad es placentera & $\square$ & $\square$ & $\square$ \\
\hline & & 6.5 & $\begin{array}{l}\text { Para mí es importante que en las relaciones sexuales exista afecto } \\
\text { y respeto }\end{array}$ & $\square$ & $\square$ & $\square$ \\
\hline \multirow{4}{*}{ G } & \multirow{4}{*}{ Movilidad } & 7.1 & $\begin{array}{l}\text { Conozco y respeto las normas de seguridad que debo tener en cuenta } \\
\text { como peatón, pasajero o conductor de bicicleta, patines, vehículo, etc. }\end{array}$ & $\square$ & $\square$ & $\square$ \\
\hline & & 7.2 & $\begin{array}{l}\text { Si monto en un vehículo como conductor o como pasajero, uso } \\
\text { el cinturón de seguridad }\end{array}$ & $\square$ & $\square$ & $\square$ \\
\hline & & 7.3 & Utilizo los puentes peatonales y las cebras & $\square$ & $\square$ & $\square$ \\
\hline & & 7.4 & $\begin{array}{l}\text { Si manejara automóvil, lo haría sin haber consumido alcohol y/o } \\
\text { sustancias psicoactivas }\end{array}$ & $\square$ & $\square$ & $\square$ \\
\hline \multirow{5}{*}{ H } & \multirow{5}{*}{$\begin{array}{c}\text { Consumo } \\
\text { de } \\
\text { sustancias }\end{array}$} & 8.1 & Evito consumir bebidas con fines energizantes & $\square$ & $\square$ & $\square$ \\
\hline & & 8.2 & Evito fumar cigarrillo & $\square$ & $\square$ & $\square$ \\
\hline & & 8.3 & Evito automedicarme & $\square$ & $\square$ & $\square$ \\
\hline & & 8.4 & Si bebo, tomo menos de cuatro tragos & $\square$ & $\square$ & $\square$ \\
\hline & & 8.5 & Evito consumir sustancias psicoactivas & $\square$ & $\square$ & $\square$ \\
\hline \multirow{5}{*}{$\mathbf{I}$} & \multirow{5}{*}{$\begin{array}{c}\text { Sentidos de } \\
\text { vida }\end{array}$} & 9.1 & Me considero una persona optimista & $\square$ & $\square$ & $\square$ \\
\hline & & 9.2 & Proyecto mis decisiones a futuro & $\square$ & $\square$ & $\square$ \\
\hline & & \begin{tabular}{|l|}
9.3 \\
\end{tabular} & Mis desiciones me acercan a lograr lo que quiero & $\square$ & $\square$ & $\square$ \\
\hline & & \begin{tabular}{|l|}
9.4 \\
\end{tabular} & Me siento satisfecho(a) conmigo y con lo que hago & $\square$ & $\square$ & $\square$ \\
\hline & & 9.5 & Sé decir NO cuando algo me molesta o incomoda & $\square$ & $\square$ & $\square$ \\
\hline \multirow{4}{*}{ J } & & 10.1 & Disfruto y cuido mi entorno & $\square$ & $\square$ & $\square$ \\
\hline & Medio & 10.2 & Reciclo & $\square$ & $\square$ & $\square$ \\
\hline & ambiente & 10.3 & Ahorro energía y agua & $\square$ & $\square$ & $\square$ \\
\hline & & 10.4 & Me reconozco como parte de la naturaleza & $\square$ & $\square$ & $\square$ \\
\hline
\end{tabular}


El descanso fue otra actividad mal evaluada por parte de los cuatros grupos poblacionales encuestados, siendo el de administrativos los que menos disfrutaban de su tiempo libre y, por ende, menos descansaban. Una hipótesis que engloba la posible explicación a las bajas calificaciones en la práctica de actividad física, nutrición y descanso, se encuentra asociada con las múltiples ocupaciones laborales que presentan los docentes y administrativos, junto con una carga académica exigente en docentes, estudiantes de pre y posgrado, que no permiten disponer del tiempo necesario para realizar actividad física frecuente, tener una nutrición balanceada y lograr un descanso reparador.

Ante estos resultados poco favorables una solución es implementar actividades educativas y estrategias tendientes a mejorar estos estilos de vida saludables, no solo en la Facultad de Medicina sino en toda la comunidad universitaria.

La sexualidad como estilo de vida saludable estuvo bien evaluada por los docentes, administrativos y estudiantes de pre y posgrado, con lo que se demostró el compromiso y el buen nivel educativo que se tenía para disfrutar de una sexualidad segura. El estudio de Profamilia "Encuesta nacional de demografía en salud" (ENSD 2010) ${ }^{4}$ demostró que el 99\% de las mujeres colombianas no unidas con vida sexual activa, utilizaban algún método para planificar y entre ellas el $86 \%$ usaba condón. Estos datos son llamativos ya que pueden diferir de la realidad; sin embargo son similares a los encontrados en nuestro estudio, en el que el 93\% de los estudiantes de pregrado y el $98 \%$ de los de posgrado se protegían ante un eventual contacto sexual. A pesar de observar un muy buen desempeño en lo concerniente a las prácticas sexuales, es imperiosa la necesidad de continuar con este tipo de educación e implementar estrategias para continuar disfrutando de una sexualidad plena y segura.
El consumo de sustancias psicoactivas no es una práctica frecuente en los integrantes de la facultad de medicina, como se demostró en nuestro estudio y al compararlo con el estudio "Caracterización epidemiológica de los estudiantes de IV año de Medicina de una universidad de Bogotá" 6 en donde el $2.6 \%$ de estudiantes consumieron sustancias psicoactivas diferentes al alcohol y al cigarrillo, lo que permite inferir un comportamiento similar en las dos poblaciones estudiadas.

\section{Conclusiones}

Aunque la escala clasifica a más del $70 \%$ de la población con hábitos saludables, el análisis de los ítems muestra inadecuados estilos de vida en relación con la actividad física, la nutrición y el descanso. A partir de esta información se deben generar estrategias de intervención colectiva que permitan modificar los factores de riesgo.

\section{Referencias}

1. Lange I, Vio F, Grunpeter H, Romo M, Castillo M, Vial B. Guía para universidades saludables y otras instituciones de educación superior. Primera edición. Santiago de Chile: Ministerio de Salud; 2006.

2. Becerra-Bulla F, Pinzón-Villate G, Vargas-Zárate M. Hacia la creación del programa universidad promotora de la salud desde la alimentación y nutrición en la Universidad Nacional de Colombia, sede Bogotá. Rev. Fac Med, 2011; 59 (supl 1):S67-79

3. Maya LH. Los estilos de vida saludables: componente de la calidad de vida. 2001. Consultada el 25 de junio de 2012. Disponible en: http://www.funlibre. org/documentos/lemaya1.htm.

4. Ojeda Gabriel. Ordoñez Myriam. Ochoa Luis Hernando .Profamilia. Encuesta nacional de la situación nutricional en Colombia 2010. En: Resumen ejecutivo ENSIN; 2010.

5. Orduz José Fernando, Ospina Juan Carlos, Beltrán Dairo "Prevalencia de los factores de riesgo cardiometabolico en la población de estudiantes de posgrado y docentes de la facultad de medicina de la Fundación Universitaria de Ciencias de la Salud 2010". (En prensa)

6. Caracterización epidemiológica de los estudiantes de IV año de medicina de una universidad de Bogotá. Medina Marcos, Murillo Luz Ángela, Gómez Heidi. 